\title{
TWO AND THREE-REVOLUTION CYCLICAL SURFACES
}

The creation of two-revolution and three-revolution cyclical surfaces is presented in the paper. Classification and vector equations of the surfaces are given. The surfaces are created by translation of the circle along the curves and its centre is on the curve. The curves are created by revolution of a point about any edge of the trihedron of the previous curve and this trihedron moves simultaneously along this curve. All specific forms of surfaces are illustrated in figures visualized in Maple.

\section{Introduction}

The point $S_{1}$ revolves about the coordinate axis $z$ with angular velocity $w_{1}$ in the distance $d_{1}$ from the origin of the coordinate system $(O, x, y, z)$. For every value of the angle $w_{1}$ there exists only one position of the point $R_{1}$ and the trajectory of this point $R_{1}$ is the curve $k_{1}$ (circle). The trihedron $\left(R_{1}, t_{1}, n_{1}, b_{1}\right)$ defined in every point $R_{1} \in k_{1}$ is determined by tangent, principal normal and binormal of the curve $k_{1}$. The point $S_{2}$ revolves at an angular velocity $w_{2}$ about any axis of the coordinate system, which is identical with the trihedron $\left(R_{1}, t_{1}, n_{1}, b_{1}\right)$ of the curve $k_{1}$, in the distance $d_{2}$ from the origin of this coordinate system which is moving simultaneously along the curve $k_{1}$. For every value of the angle $w_{2}$ there exists only one position of the point $R_{2}$. The trajectory of this point $R_{2}$ is the curve $k_{2}^{g}$, where $g=t, n, b$. The trihedron $\left(R_{2}\right.$, $t_{2}, n_{2}, b_{2}$ ) in every point $R_{2} \in k_{2}^{g}$ is determined by the tangent, principal normal and binormal of the curve $k_{2}^{g}$. The point $S_{3}$ revolves about any axis of the coordinate system identical with the trihedron $\left(R_{2}, t_{2}, n_{2}, b_{2}\right)$ of the curve $k_{2}^{g}$ at an angular velocity $w_{3}$ in the distance $d_{3}$ from the origin of this coordinate system which is moving simultaneously along the curve $k_{2}^{g}$. For every value of the angle $w_{3}$ there exists only one position of the point $R_{3}$. The trajectory of the point $R_{3}$ is the curve $k_{3}^{g h}$, where $g, h=t, n, b$. The trihedron $\left(R_{3}, t_{3}, n_{3}, b_{3}\right)$ in every point $R_{3} \in k_{3}^{g h}$ is determined by the tangent, principal normal and binormal of the curve $k_{3}^{g h}$.

The surface of the type $P_{1}(u, v)$ is created by translation of the circle $c_{1}=\left(R_{1}, r_{1}\right)$ along the curve $k_{1}$, the surface of the type $P_{2}^{g}(u, v)$ is created by translation of the circle $c_{2}=\left(R_{2}, r_{2}\right)$ along the curve $k_{2}^{g}$ and the surface of the type $P_{3}^{g h}(u, v)$ is created by translation of the circle $c_{3}=\left(R_{3}, r_{3}\right)$ along the curve $k_{3}^{g h}$. The index $g=t, n, b$ determines that the point $S_{2}$ revolves about the tangent $t_{1}$, or principal normal $n_{1}$ or binormal $b_{1}$ of the curve $k_{1}$ and the index $h=t, n, g$ determines that the point $S_{3}$ revolves about tangent $t_{2}$, principal normal $n_{2}$ or binormal $b_{2}$ of the curve $k_{2}^{g}$.

\section{Vector functions of the curves $k_{1}, k_{2}^{g}, k_{3}^{g h}$}

Let the curve $k_{1}$ be a circle created by revolution of the point $S_{1}=S_{1}\left(d_{1}, 0,0,1\right)$ about the axis $z$ of the coordinate system $(O$, $x, y, z)$ at an angular velocity $w_{1}=v$ and $k_{1}$ is determined by the vector function

$$
\begin{aligned}
& r_{1}(v)=\left(x_{k 1}(v), y_{k 1}(v), z_{k 1}(v), 1\right)=S_{1} \cdot T_{z 1}\left(w_{1}\right)= \\
& =\left(d_{1} \cos v, s_{1} q_{1} \sin v, 0,1\right), v \in\langle 0,2 \pi\rangle .
\end{aligned}
$$

The matrix $T_{z 1}\left(w_{1}\right)$ represents the revolution of the point $S_{1}$ about the coordinate axis $z$ given by (5) ( $3^{\text {rd }}$ matrix for $i=1$ ), where the parameter $q_{1}= \pm 1$ determines the right-turned or leftturned revolution movement of the point (Fig. 1, $i=1, j=z$ ) [3]. We will define the trihedron $\left(R_{1}, t_{1}, n_{1}, b_{1}\right)$ of the curve $k_{1}$ in every point $R_{1} \in k_{1}$ by the tangent $t_{1}$, principal normal $n_{1}$ and by binormal $b_{1}$ with their unit vectors $t_{1}(v), n_{1}(v), b_{1}(v)$ by equations (2), (3), (4) for $i=1, v \in\langle 0,2 \pi\rangle$

$$
\begin{aligned}
& t_{1}(v)=\left(a_{t i}, b_{t i}, c_{t i}\right)=\frac{1}{h_{1 i}} \frac{d r_{1}}{d v}= \\
& =\frac{1}{h_{1 i}}\left(\frac{d x_{k i}(v)}{d v}, \frac{d y_{k i}(v)}{d v}, \frac{d z_{k i}(v)}{d v}\right), h_{1 i}=\left|\frac{d r_{1}}{d v}\right| \\
& n_{1}(v)=\left(a_{n i}, b_{n i}, c_{n i}\right)=\frac{1}{h_{2 i}} \frac{d^{2} r_{1}}{d v^{2}}= \\
& =\frac{1}{h_{2 i}}\left(\frac{d^{2} x_{k i}(v)}{d v^{2}}, \frac{d^{2} y_{k i}(v)}{d v^{2}}, \frac{d^{2} z_{k i}(v)}{d v^{2}}\right), h_{2 i}=\left|\frac{d^{2} r_{1}}{d v^{2}}\right| \\
& b_{i}=\frac{1}{h_{3 i}}\left(t_{i}(v) \times n_{i}(v)\right), h_{3 i}=\left|t_{i}(v) \times n_{i}(v)\right| .
\end{aligned}
$$

The curve $k_{2}^{g}$ is created by revolution of the point $S_{2}$ in the distance $d_{2}$ from the origin of the coordinate system $(O, x, y, z)$ about any coordinate axis $x, y$, or $z$ through the angle $w_{2}$ into the point $S_{2}^{\prime}$ (Fig. $1, i=2$ for , $j=x, y, z$ ). Angular velocity $w_{2}=m_{1} v$

\footnotetext{
* Tatiana Olejnikova

Institute of Technology, Economics and Management in Civil Engineering, Civil Engineering Faculty, Technical University of Kosice, Slovakia, E-mail: tatiana.olejnikova@tuke.sk
} 

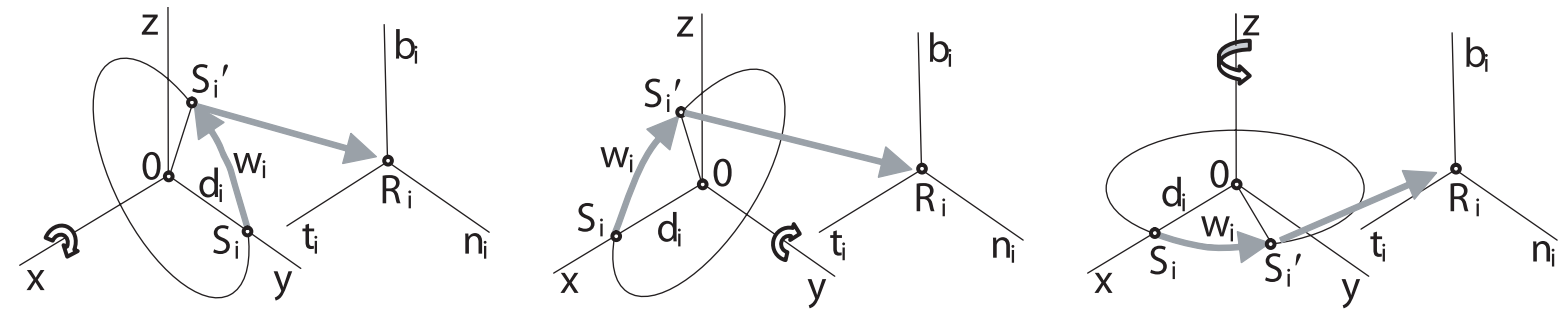

Fig. 1 Revolution of the point about axes $x, y, z$

of the point $S_{2}$ is $m_{1}$-multiple of angular velocity $w_{1}=v$ of the point $S_{1}$ The point $S_{2}^{\prime}$ is transformed into the point $R_{2}$ in the coordinate system $\left(R_{1}, t_{1}, n_{1}, b_{1}\right)$. If we create a surface of the type $P_{2}^{g}(u, v)$, where $g=t$ (or $g=n$, or $g=b$ ), we will revolve the point $S_{2}$ about the axis $j=x$, or $j=y$, or $j=z$. The revolution of the point $S_{2}$ is represented by a matrix $T_{j 2}\left(w_{2}\right) . j=x, y, z$ in (5), where the parameter $q_{2}= \pm 1$ determines the right-turned or left-turned revolution and the transformation of the point $S_{2}^{\prime}$ into the point $R_{2}$ is represented by a matrix $M_{2}\left(w_{2}\right)$ given by (5) [4]. The point $S_{2}$. will be situated always on any coordinate axis $x, y, z$.

$$
\begin{aligned}
T_{x i}\left(w_{i}\right) & =\left(\begin{array}{cccc}
1 & 0 & 0 & 0 \\
0 & \cos w_{i} & q_{i} \sin w_{i} & 0 \\
0 & -q_{i} \sin w_{i} & \cos w_{i} & 0 \\
0 & 0 & 0 & 1
\end{array}\right), \\
T_{z i}\left(w_{i}\right) & =\left(\begin{array}{cccc}
\cos w_{i} & 0 & q_{i} \sin w_{i} & 0 \\
0 & 1 & 0 & 0 \\
-q_{i} \sin w_{i} & 0 & \cos w_{i} & 0 \\
0 & 0 & 0 & 1
\end{array}\right), \\
T_{z i}\left(w_{i}\right) & =\left(\begin{array}{cccc}
\cos w_{i} & q_{i} \sin w_{i} & 0 & 0 \\
-q_{i} \sin w_{i} & \cos w_{i} & 0 & 0 \\
0 & 0 & 1 & 0 \\
0 & 0 & 0 & 1
\end{array}\right), \\
M_{i}\left(w_{i}\right) & =\left(\begin{array}{llll}
a_{i i} & b_{i i} & c_{i i} & 0 \\
a_{n i} & b_{n i} & c_{n i} & 0 \\
a_{b i} & b_{b i} & c_{b i} & 0 \\
0 & 0 & 0 & 1
\end{array}\right) .
\end{aligned}
$$

The elements of the matrix $M_{1}\left(w_{1}\right)$ in (5) are the coordinates of unit vectors $t_{i}(v), n_{i}(v), b_{i}(v)$ of tangent $t_{i}$, principal normal $n_{i}$ and binormal $b_{i}$ in trihedron $\left(R_{i}, t_{i}, n_{i}, b_{i}\right), i=1,2,3$. Then the vector function of the curve $k_{2}^{g}$ is

$$
\begin{aligned}
& r_{2}(v)=\left(x_{k 2}(v), y_{k 2}(v), z_{k 2}(v), 1\right)=S_{1} \cdot T_{z 1}\left(w_{1}\right)+ \\
& +S_{2} \cdot T_{j 2}\left(w_{2}\right) \cdot M_{2}\left(w_{2}\right), j=x, y, z
\end{aligned}
$$

and $S_{2}=S_{2}\left(d_{2}, 0,0,1\right)$ or $S_{2}=S_{2}\left(0,0, d_{2}, 1\right)$.

The trihedron $\left(R_{2}, t_{2}, n_{2}, b_{2}\right)$ is determined in every point $R_{2}$ $\in k_{2}^{g}$ by the tangent $t_{2}$, principal normal $n_{2}$ and binormal $b_{2}$ with the unit vectors $t_{2}(v), n_{2}(v), b_{2}(v)$ expressed by equations (2), (3), (4) for $i=2$.
The curve $k_{3}^{g h}$ is created by revolution of the point $R_{3}$ in the distance $d_{3}$ from the origin of the coordinate system $(O, x, y, z)$ about any coordinate axis $x, y$, or $z$ through the angle $w_{3}$ into the point $S_{3}^{\prime}$ (Fig. 2, $i=3$ for, $j=x, y, z$ ). Angular velocity $w_{3}=$ $=m_{2} w_{2}=m_{2} m_{1} v$ of the point $S_{3}$ is $m_{2}$-multiple of angular velocity $w_{2}=m_{1} v$ of the point $S_{2}$. The point $S_{3}^{\prime}$ is transformed into the point $R_{3}$ in the coordinate system $\left(R_{2}, t_{2}, n_{2}, b_{2}\right)$. If we create the surface of type $P_{3}^{g h}(u, v)$, where $h=t$ ( or $h=n$, or $h=b$ ), we will revolve the point $S_{3}$ about the axis $j=x$, or $j=y$, or $j=z$. The revolution of the point $S_{3}$ is represented by a matrix $T_{j 3}\left(w_{3}\right), j=$ $=x, y, z$, where the parameter $q_{3}= \pm 1$ determines the rightturned or left-turned revolution and transformation of the point $S_{3}^{\prime}$ into the point $R_{3}$ is represented by the matrix $M_{3}\left(w_{3}\right)$ by equations (5) [4]. The point $S_{3}$.will always be situated on any coordinate axis $x, y$, or $z$.

Then the vector function of the curve $k_{3}^{g h}$ for $j=x, y, z$ is

$$
\begin{aligned}
& r_{3}(v)=\left(x_{k 3}(v), y_{k 3}(v), z_{k 3}(v), 1\right)=S_{1} \cdot T_{z 1}\left(w_{1}\right)+ \\
& +S_{2} \cdot T_{j 2}\left(w_{2}\right) \cdot M_{2}\left(w_{2}\right)+S_{3} \cdot T_{j 3}\left(w_{3}\right) \cdot M_{3}\left(w_{3}\right), \\
& j=x, y, z .
\end{aligned}
$$

The trihedron $\left(R_{3}, t_{3}, n_{3}, b_{3}\right)$ in every point $R_{3} \in k_{3}^{g h}$ is determined by the tangent $t_{3}$, principal normal $n_{3}$ and binormal $b_{3}$ with the unit vectors $t_{3}(v), n_{3}(v), b_{3}(v)$ by equations (2), (3), (4) for $i=3$.

In Fig. 2 there is displayed a revolution of the point $S_{1}$ about the coordinate axis $z$ through the angle $w_{1}$ into the point $R_{1}$, where its revolutionary movement creates the curve $k_{1}$, revolution of the point $S_{2}$ about the coordinate axis $z$ through the angle $w_{2}$ into the point $S_{2}^{\prime}$ and its transformation into the point $R_{2}$, where its revolutionary movement creates the curve $k_{2}^{g}$, revolution of the point $S_{3}$ about the coordinate axis $z$ through the angle $w_{3}$ into the point $S_{3}^{\prime}$ and its transformation into the point $R_{3}$, where its revolutionary movement creates the curve $k_{3}^{g h}$. In the points $R_{1}, R_{2}, R_{3}$ there are displayed trihedrons $\left(R_{1}, t_{1}, n_{1}, b_{1}\right),\left(R_{2}, t_{2}, n_{2}, b_{2}\right),\left(R_{3}, t_{3}, n_{3}\right.$, $\left.b_{3}\right)$.

In Fig. 3 there are displayed for illustration only three combinations of the curves $k_{1}, k_{2}^{t}, k_{3}^{t t}, k_{1}, k_{2}^{n}, k_{3}^{n n}$ and $k_{1}, k_{2}^{b}, k_{3}^{b b}$. 


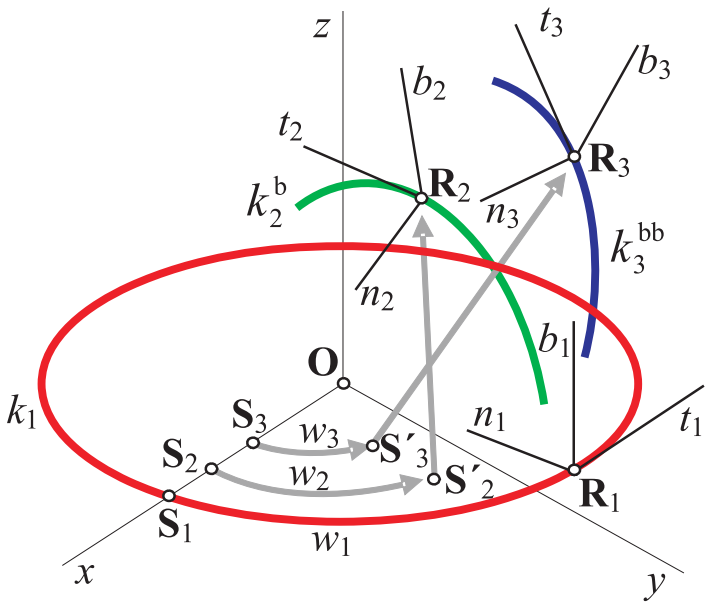

Fig. 2 Creation the curves $k_{1}, k_{2}^{g}, k_{3}^{g h}$ and their trihedrons

The two-revolution cyclical surface of the type $P_{2}^{g}(u, v)$ is created by translation of the circle $c_{2}=\left(R_{2}, r_{2}\right)$ along the curve $k_{2}^{g}$ at an angular velocity $w_{2}=m_{1} v$, where the circle is always in the plane $\left(n_{2}, b_{2}\right)$ if the index $g=t$, or in the plane $\left(t_{2}, b_{2}\right)$ if $g=n$, or in the plane $\left(t_{2}, n_{2}\right)$ if $g=b$ and its centre is the point $R_{2} \in k_{2}^{g}$. We will create it so that the circle $c_{02}$ determined by the vector function $c_{02}(u)=\left(0, r_{2} \cos u, r_{2} \sin u, 1\right)$ if $g=t$, or $c_{02}(u)=\left(r_{2} \cos u\right.$, $\left.0, r_{2} \sin u, 1\right)$ if $g=n$, or $c_{02}(u)=\left(r_{2} \cos u, r_{2} \sin u, 0,1\right)$ if $g=b$ we will transform into the circle $c_{2}$ in the coordinate system $\left(R_{2}\right.$, $t_{2}, n_{2}, b_{2}$ ) using the matrix $M_{2}\left(w_{2}\right)$ by equations (5) (Fig. 4). The vector function of the cyclical surface of the type $P_{2}^{g}(u, v)$ is

$$
\begin{aligned}
& P_{2}^{g}(u, v)=r_{2}(v)+c_{02}(u) \cdot M_{2}\left(w_{2}\right), \\
& u \in\langle 0,2 \pi\rangle . v \in\langle 0,2 \pi\rangle .
\end{aligned}
$$

where $r_{2}(v)$ is the vector function of the curve $k_{2}^{g}$ determined by (6).
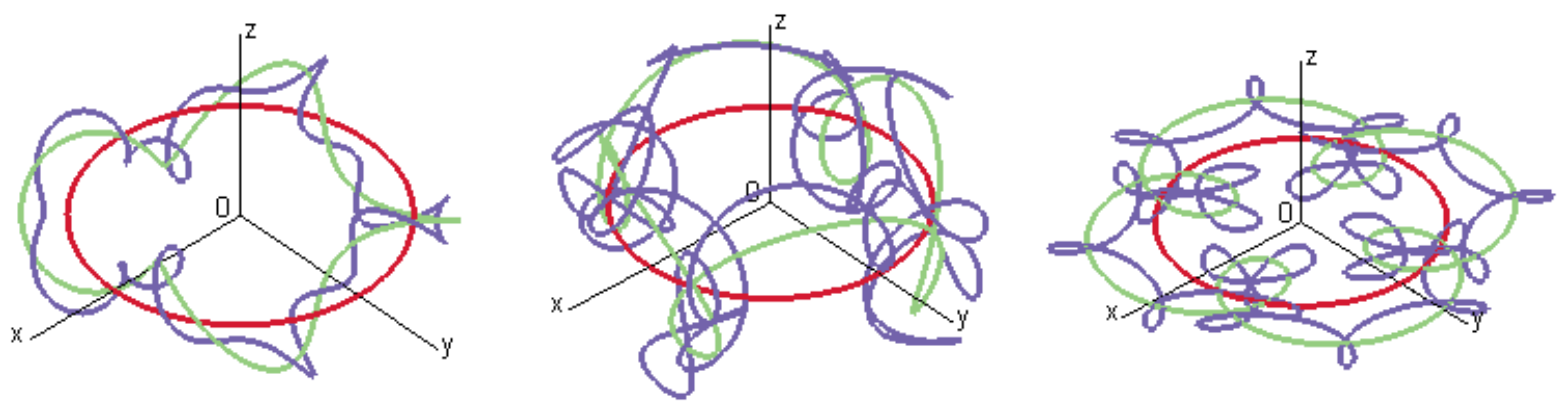

Fig. 3 Combinations of the curves $k_{1}, k_{2}^{t}, k_{3}^{t t}, k_{1}, k_{2}^{n}, k_{3}^{n n}$ and $k_{1}, k_{2}^{b}, k_{3}^{b b}$.

\section{Vector functions of cyclical surfaces of the type $P_{1}(u, v), P_{2}^{g}(u, v), P_{3}^{g h}(u, v)$}

The cyclical surface of the type $P_{1}(u, v)$ is created by translation of the circle $c_{1}=\left(R_{1}, r_{1}\right)$ in the plane $\left(n_{1}, b_{1}\right)$ along the curve $k_{1}$ at an angular velocity $w_{1}=v$. We will create it so that the circle $c_{01}$ determined by the vector function $c_{01}(u)=\left(0, r_{1} \cos u, r_{1} \sin u\right.$, 1) will be transformed into the circle $c_{1}$ in the coordinate system $\left(R_{1}, t_{1}, n_{1}, b_{1}\right)$ using the matrix $M_{1}\left(w_{1}\right)$ expressed by equations (5) (Fig. 4).

The vector function of the cyclical surface of the type $P_{1}(u, v)$ is

$$
\begin{aligned}
& P_{1}(u, v)=r_{1}(v)+c_{01}(u) \cdot M_{1}\left(w_{1}\right), \\
& u \in\langle 0,2 \pi\rangle . v \in\langle 0,2 \pi\rangle .
\end{aligned}
$$

where $r_{1}(v)$ is the vector function of the curve $k_{1}$ determined by equation (1). This surface is surface of torus.
The three-revolution cyclical surface of the type $P_{3}^{g h}(u, v)$ is created by translation of the circle $c_{3}=\left(R_{3}, r_{3}\right)$ along the curve $k_{3}$ at an angular velocity $w_{3}=m_{2} m_{1} v$, where the circle is always in the plane $\left(n_{3}, b_{3}\right)$ if the index $h=t$, or in the plane $\left(t_{3}, b_{3}\right)$ if $h=n$, or in the plane $\left(t_{3}, n_{3}\right)$ if $h=b$ and its centre is the point $R_{3} \in k_{3}^{g h}$. We will create it so that the circle $c_{03}$ determined the by vector function $c_{03}(u)=\left(0, r_{3} \cos u, r_{3} \sin u, 1\right)$ if $h=t$, or $c_{03}(u)=\left(r_{3} \cos u, 0, r_{3} \sin u, 1\right)$ if $h=n$, or $c_{03}(u)=\left(r_{3} \cos u, r_{3}\right.$ $\sin u, 0,1)$ if $h=b$ we will transform into the circle $c_{3}$ in the coordinate system $\left(R_{3}, t_{3}, n_{3}, b_{3}\right)$ using the matrix $M_{3}\left(w_{3}\right)$ by equations (5). The vector function of the cyclical surface of the type $P_{3}^{g h}(u, v)$ is

$$
\begin{aligned}
& P_{3}^{g h}(u, v)=r_{3}(v)+c_{03}(u) \cdot M_{3}\left(w_{3}\right), \\
& u \in\langle 0,2 \pi\rangle . v \in\langle 0,2 \pi\rangle .
\end{aligned}
$$

where $r_{3}(v)$ is the vector function of the curve $k_{3}^{g h}$ determined by (7). 

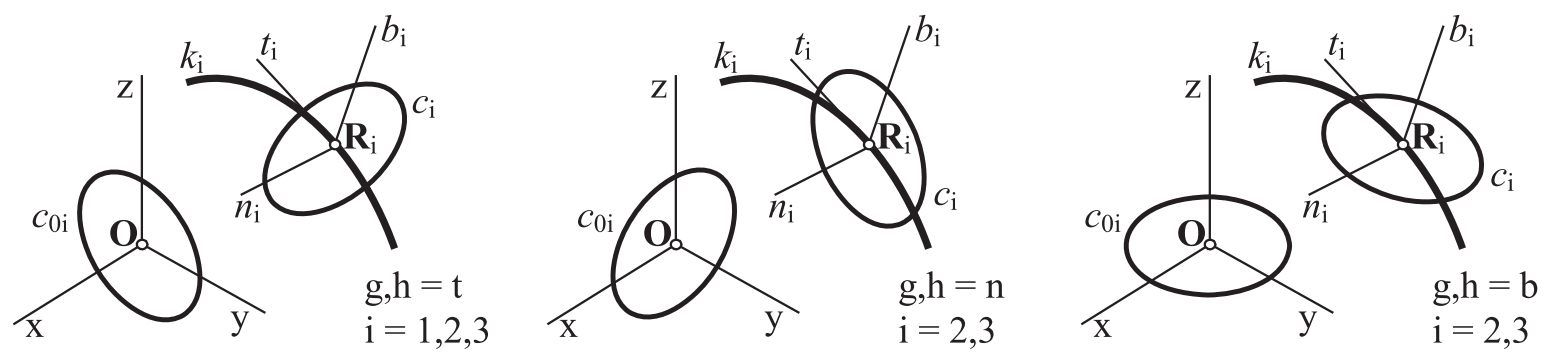

Fig. 4 Transformation of the circle $c_{0 i}$ into the circle $c_{i}$

\section{Classification of cyclical surfaces of the type} $P_{2}^{g}(u, v), P_{3}^{g h}(u, v)$

The two-revolution cyclical surface of the type $P_{2}^{g}(u, v)$ can be classified according to the index $g$ :

Table 1: Classification of cyclical surfaces of the type $P_{2}^{g}(u, v)$

\begin{tabular}{|c|c|c|c|}
\hline $\boldsymbol{g}$ & $\boldsymbol{t}$ & $\boldsymbol{n}$ & $\boldsymbol{b}$ \\
\hline & $P_{2}^{t}(u, v)$ & $P_{2}^{n}(u, v)$ & $P_{2}^{b}(u, v)$ \\
\hline
\end{tabular}

The three-revolution cyclical surface of the type $P_{3}^{g h}(u, v)$ can be classified according to the index $g$ and $h$ :

Table 2: Classification of cyclical surfaces of the type $P_{3}^{g h}(u, v)$

\begin{tabular}{|c|c|c|c|}
\hline $\boldsymbol{g} / \boldsymbol{h}$ & $\boldsymbol{t}$ & $\boldsymbol{n}$ & $\boldsymbol{b}$ \\
\hline $\boldsymbol{t}$ & $P_{3}^{t t}(u, v)$ & $P_{3}^{t n}(u, v)$ & $P_{3}^{t b}(u, v)$ \\
\hline $\boldsymbol{n}$ & $P_{3}^{n t}(u, v)$ & $P_{3}^{n n}(u, v)$ & $P_{3}^{n b}(u, v)$ \\
\hline $\boldsymbol{b}$ & $P_{3}^{b t}(u, v)$ & $P_{3}^{b n}(u, v)$ & $P_{3}^{b b}(u, v)$ \\
\hline
\end{tabular}

\section{Illustrations of cyclical surfaces of the type $P_{1}(u, v), P_{2}^{g}(u, v), P_{3}^{g h}(u, v)$}

In Fig. 5 there are displayed three combinations of cyclical surfaces of the type $P_{1}(u, v), P_{2}^{t}(u, v), P_{3}^{t t}(u, v)$ in fig. a), $P_{1}(u, v)$, $P_{2}^{t}(u, v), P_{3}^{t n}(u, v)$ in fig.b), $P_{1}(u, v), P_{2}^{t}(u, v), P_{3}^{t b}(u, v)$ in fig. c).

In Fig. 6 there are displayed three combinations of cyclical surfaces of the type $P_{1}(u, v), P_{2}^{n}(u, v), P_{3}^{n t}(u, v)$ in fig. a), $P_{1}(u, v)$, $P_{2}^{n}(u, v), P_{3}^{n n}(u, v)$ in fig. b), $P_{1}(u, v), P_{2}^{n}(u, v), P_{3}^{n b}(u, v)$ in fig. c).

In Fig. 7 there are displayed three combinations of cyclical surfaces of the type $P_{1}(u, v), P_{2}^{b}(u, v), P_{3}^{b t}(u, v)$ in fig. a), $P_{1}(u, v)$, $P_{2}^{b}(u, v), P_{3}^{b n}(u, v)$ in fig.b), $P_{1}(u, v), P_{2}^{b}(u, v), P_{3}^{b b}(u, v)$ in fig. c).

The surfaces mentioned above can be used in design practice as constructive or ornamental structural components.

The paper was supported by the grant VEGA 1 / 4002 / 07.

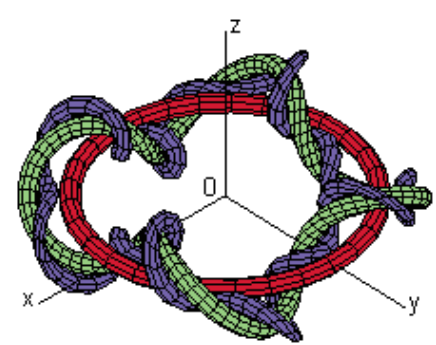

a)

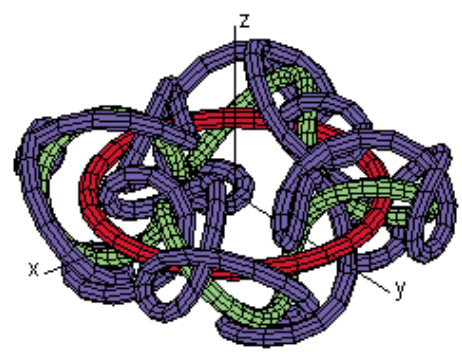

b)

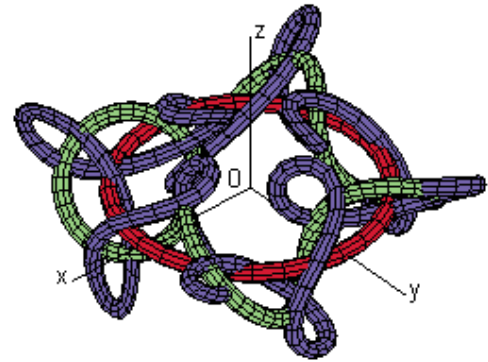

c)

Fig. 5 Combinations of cyclical surfaces for $g=t$ 


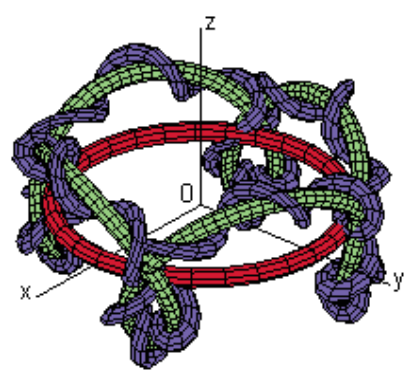

a)

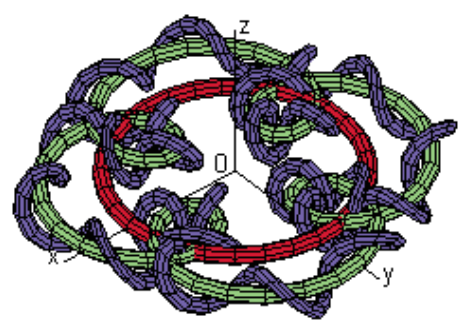

a)

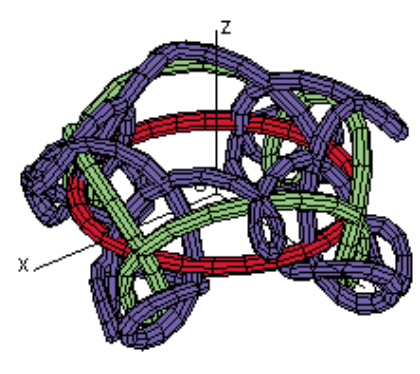

b)

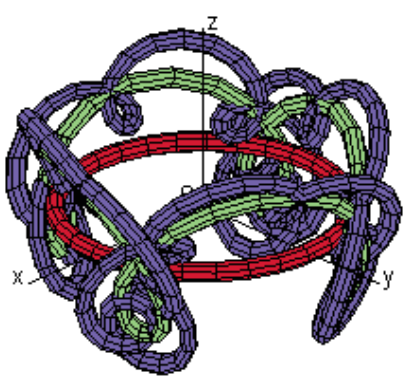

c)

Fig. 6 Combinations of cyclical surfaces for $g=n$

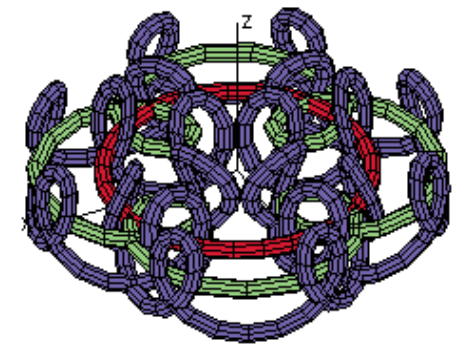

b)

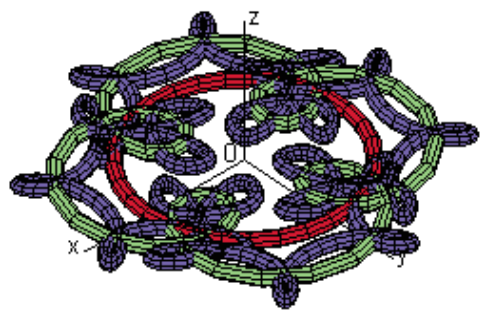

c)

Fig 7 Combinations of cyclical surfaces for $g=b$

\section{References}

[1] OLEJNIKOVA, T.: Straight-lines Spiral-rotary Planes (in Slovak), Proc. of 26. konference o geometrii a pocitacove grafice, 2006, Nove Mesto na Morave, CR, 2006, p. 15-28, ISBN 80-7040-902-9.

[2] OLEJNIKOVA, T.: Straight-lines Spiral-rotary Planes II (in Slovak), Proc. of 6th International Conference APLIMAT 2007, Bratislava, 2007, p. 231-236, ISBN 978-80-969562-4-1

[3] KOHOUT, V.: Differential Geometry (in Czech), SNTL-Publishers of Technical Literature, Praha, 1971.

[4] GRANAT, L., SECHOVSKY, H.: Computer Graphics (in Slovak), SNTL - Publishers of Technical Literature, Bratislava, 1974 